\title{
AIP The Journal of Chemical Physics
}

Nonadiabatic approach for resonant molecular multiphoton absorption processes in intense infrared laser fields

TakSan Ho and Shihl Chu

Citation: The Journal of Chemical Physics 79, 4708 (1983); doi: 10.1063/1.445612

View online: http://dx.doi.org/10.1063/1.445612

View Table of Contents: http://scitation.aip.org/content/aip/journal/jcp/79/10?ver=pdfcov

Published by the AIP Publishing

Articles you may be interested in

Self-interaction-free time-dependent density-functional theory for molecular multiphoton processes in intense laser fields

AIP Conf. Proc. 525, 415 (2000); 10.1063/1.1291959

Quasiperiodic and chaotic motions in intense field multiphoton processes

AIP Conf. Proc. 160, 282 (1987); 10.1063/1.36851

A study of multiphoton resonances in $\mathrm{Kr}$ and $\mathrm{Ar}$ under intense laser field

AIP Conf. Proc. 160, 243 (1987); 10.1063/1.36840

The enhancement of intense laser induced multiphoton processes by a static electric field

J. Chem. Phys. 79, 4912 (1983); 10.1063/1.445583

Multiphoton molecular dissociation in intense laser fields

J. Chem. Phys. 65, 5204 (1976); 10.1063/1.433073

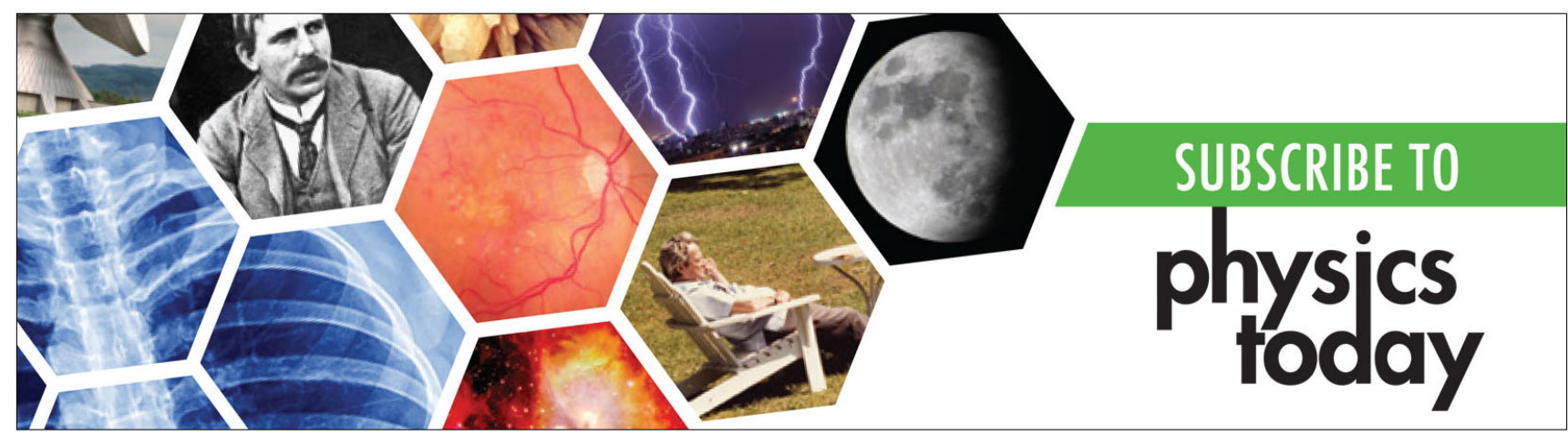




\title{
Nonadiabatic approach for resonant molecular multiphoton absorption processes in intense infrared laser fields
}

\author{
Tak-San Ho and Shih-I Chua) \\ Department of Chemistry, University of Kansas, Lawrence, Kansas 66045 \\ (Received 14 July 1983; accepted 9 August 1983)

\begin{abstract}
A nonperturbative approach for efficient and accurate treatment of the molecular multiphoton absorption (MPA) quantum dynamics in intense infrared (IR) laser fields is presented. The approach is based on the adiabatic separation of the fast vibrational motion from the slow rotational motion, incorporating the fact that the IR laser frequency is close to the frequencies of adjacent vibrational transitions. One thus first solves the quasivibrational energy (QVE) states (or, equivalently, the dressed vibrational states) with molecular orientation fixed. This reduces the computationally often formidable (vibrational-rotational) Floquet matrix analysis to a manageable scale, and, in addition, provides useful physical insights for understanding the nonlinear MPA dynamics. The QVE levels are found to be grouped into distinct energy bands, characterized by the IR frequency, with each band providing an effective potential for molecular rotation. Whereas the interband couplings are totally negligible, the intraband nonadiabatic angular couplings are the main driving mechanisms for inducing resonant vibrational-rotational multiphoton transitions. The utility of the method is illustrated by a detailed study of the sequential MPA spectra for ${ }^{12} \mathrm{C}^{16} \mathrm{O}$ molecule, including state-to-state multiquantum transitions and transitions from initially thermally distributed states as a whole. Results are presented for the case of IR laser intensity $50 \mathrm{GW} / \mathrm{cm}^{2}$ and frequencies ranging from 2115 to $2165 \mathrm{~cm}^{-1}$. Excellent agreement of the MPA spectra obtained by the nonadiabatic approach and the exact Floquet matrix method was observed in all fine details.
\end{abstract}

\section{INTRODUCTION}

There is currently much interest in the study of the collisionless multiphoton excitation (MPE) and dissociation (MPD) of polyatomic molecules in the presence of intense, pulsed, infrared (IR) lasers. ${ }^{1}$ Although the qualitative features of the MPE/MPD processes appear to be established, ${ }^{1}$ quantitative analysis of the underlying quantum dynamics remains to be a major challenge. The main obstacle stems from the extreme complexity of the molecular level structure. In particular, the combined motion of vibration and rotation leads to a great wealth of possible multiphoton transition pathways, rendering the problem to be a formidable one.

From the theoretical point of view, the semiclassical Floquet theory ${ }^{2,3}$ has provided a powerful $a b$ initio technique for nonperturbative treatment of $\mathrm{MPE}^{4,5}$ and $M P D^{6}$ of some simple systems. In practice, the Floquet matrix is truncated to $N$ by $N$ in dimension with $N=N_{F} N_{V} N_{J}$, where $N_{F}$ is the number of Floquet photon blocks, $N_{V}$ the number of vibrational levels included in one Floquet block, and $N_{J}$ the number of rotational states included in one vibrational level. As $N$ increases rapidly with the size of the molecule, and as the computational expense grows as $N^{3}$, the full Floquet analysis can become prohibitively costly even for simple diatomic molecules. We are thus led to seek new approximate techniques capable of providing accurate results yet involving much smaller Floquet matrix manipulations. The situation here is analogous to the field of molecular collision theory: Where a variety of angular momentum decoupling methods ${ }^{7}$ have been developed in the last decade to alleviate the problem of a large number of coupled equations.

${ }^{2}$ Alfred P. Sloan Foundation Fellow.
In the process of IR multiphoton absorption (MPA), the laser frequency is of the same order of magnitude as the molecular vibrational frequency and considerably exceeds the rotational frequency. More explicitly, since the Hamiltonian contains fast (vibrational motion and electric dipole interaction) and slow (rotational motion) parts, one anticipates the wave function will have the adiabatic form of the product of a rapidly oscillating function times a function slowly varying in time. One can determine the rapidly oscillating part by considering the Schrödinger equation without the rotational Hamiltonian. This gives rise to an effective quasivibrational energy (QVE) which depends parametrically on the orientation of the molecule. The slowly varying parts of the wave function can then be solved by using the effective QVE as the potential energy for the rotational motion. This adiabatic picture has been used previously ${ }^{8}$ to obtain approximate analytic solutions for the rotational spectra of diatomic molecules in some special cases. It provides an adequate zeroth order description and a convenient basis for our development of an economical and accurate approach for MPA studies. As will be shown later, however, the adiabatic approximation tends to break down nearby resonant transition regions and nondiabatic angular couplings between nearly degenerate adiabatic QVE states are required for a proper MPA analysis.

Preliminary discussion of this idea has been given in a previous communication. ${ }^{\theta}$ In this paper, we provide a more detailed account of the theory, the numerical methodas well as the analysis of nonlinear MPA quantum. dynamics. After a brief introduction to the conventional Floquet Hamiltonian method in Sec. II A, we discuss the theoretical formulation of the nondiabatic approach for the case of diatomic molecules in Sec. II B. Extension to polyatomics is straightforward. In Sec. III, we provide a detailed numerical analysis of the resonant MPA 
process in ${ }^{12} \mathrm{C}^{16} \mathrm{O}$. This is followed by a conclusion in Sec. IV.

\section{THEORY}

Consider the vibrational-rotational motion of a heteronuclear diatomic molecule in the presence of a coherent monochromatic field $\mathbf{E}=\mathbf{E}_{0} \cos \omega t$ of an IR laser. The Schrödinger equation for the system in the electric dipole approximation (in a. u. ) is

$$
i \partial \Psi(\mathbf{r}, t) / \partial t=\hat{H}(\mathbf{r}, t) \Psi(\mathbf{r}, t),
$$

where

$$
\hat{H}(\boldsymbol{r}, t)=\hat{H}_{v}^{(0)}(r)+\hat{H}_{R R}(\hat{r})-\mu(r) E_{0} \cos \theta \cos \omega t .
$$

Here $\hat{H}_{v}^{(0)}(r)$ is the diatom vibrational Hamiltonian [with eigenfunctions $\zeta_{v}(r)$ and eigenvalues $\left.E_{v}^{(0)}\right]$ and $\hat{H}_{R R}(\hat{r})$ is the rigid rotator Hamiltonian [with spherical harmonics eigenfunctions $Y_{j m_{j}}(\hat{r}=\theta \phi)$ and eigenvalues $B_{\alpha} j(j+1)$, where $j$ and $m_{j}$ are, respectively, the rotational angular momentum and its projection quantum number and $B_{e}$ is the rotational constant]. The vibrational-rotational interaction has been ignored for simplicity but can be incorporated if necessary. $\mu(r)$ is the molecular electric dipole moment and $\theta$ is the angle between the direction of the field $\left(E_{0}|| z\right)$ and the axis of the molecule. As the interaction energy in $\mathrm{Eq} .(2)$ is independent of the azimuthal angle $\phi, m_{f}$ is a constant of motion.

\section{A. An outline of Floquet theory and quasienergy method}

Since the total Hamiltonian $H(\mathbf{r}, t)[\mathrm{Eq} .(2)]$ is periodic in time, $H(r, t)=H(\mathbf{r}, t+T), T=2 \pi / \omega$, the Floquet theorem ${ }^{2,3,10}$ admits the solution of Eq. (1) in the following form:

$$
\Psi_{e_{\beta}}(\mathbf{r}, t)=\exp \left(-i \epsilon_{\beta} t\right) \psi_{\varepsilon_{\beta}}(\mathbf{r}, t),
$$

where $\Psi_{\epsilon_{B}}$ is called the quasienergy state (QES) corresponding to the quasienergy $\epsilon_{\beta}$ and $\psi_{\epsilon_{\beta}}$ is a periodic function of time, viz.

$$
\psi_{\epsilon_{B}}(\mathbf{r}, t+T)=\psi_{\epsilon_{B}}(r, t), \quad T=2 \pi / \omega .
$$

The wave functions $\left\{\psi_{\epsilon_{\beta}}(r, t)\right\}$ form a complete orthonormal basis in the Hilbert space and are solutions of the following eigenvalue equation:

$$
[\hat{H}(\mathbf{r}, t)-i \partial / \partial t] \psi_{\epsilon_{\beta}}(\mathbf{r}, t)=\epsilon_{\beta} \psi_{\epsilon_{\beta}}(\mathbf{r}, t) .
$$

Following the Floquet Hamiltonian method, ${ }^{2,5}$ one can reduce the dynamic problem [Eq. (5)] into an equivalent static problem by expanding $\psi_{\epsilon_{\beta}}$ into a Fourier series (indexed by $n$ ),

$$
\left.\psi_{\epsilon_{\beta}}(\mathbf{r}, t)=\sum_{n=-\infty}^{\infty} \exp (i n \omega t) \sum_{\nu} \sum_{j=\left\{m_{j} j\right.}\langle v j, n| \epsilon_{\beta}\right) \zeta_{v}(r) Y_{j m_{j}}(\theta, \phi) .
$$

The expansion coefficients $\left\langle v j, n \mid \epsilon_{\beta}\right\rangle$ in Eq. (6) can be determined from the solution of the following Floquet matrix eigenvalue equation ${ }^{5}$

$\sum_{v^{\prime}} \sum_{j^{\prime}} \sum_{n^{\prime}}\left\langle v j, n\left|\hat{H}_{F}\right| v^{\prime} j^{\prime}, n^{\prime}\right\rangle\left\langle v^{\prime} j^{\prime}, n^{\prime} \mid \epsilon_{B}\right\rangle=\epsilon_{B}\left\langle v j, n \mid \epsilon_{B}\right\rangle$,

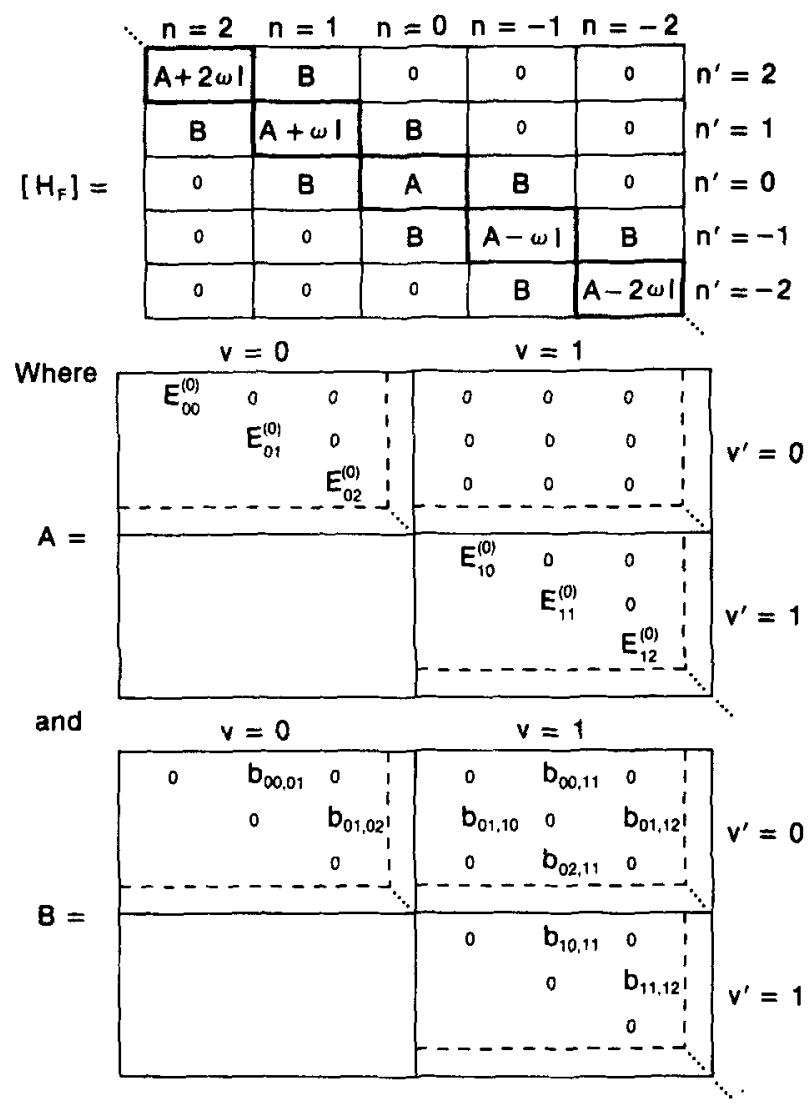

FIG. 1. Structure of the exact Floquet Hamiltonian $H_{F}$ in the Floquet state basis $\{|v j, n\rangle\}$. The Hamiltonian is composed of the diagonal Floquet blocks of type $A$, and the off-diagonal blocks of type $B . E_{v j}^{(0)}$ are the unperturbed vibrational-rotational energies, and $b_{v f, v^{\circ} j^{\prime}}$ are electronic dipole coupling matrix elements.

$$
\begin{gathered}
\left\langle v j, n\left|\hat{H}_{F}\right| v^{\prime} \jmath^{\prime}, n^{\prime}\right\rangle=\left\langle v j\left|\hat{H}_{F}^{\left(n-n^{\prime}\right)}(\mathbf{r})\right| v^{\prime} j^{\prime}\right\rangle+n \omega \delta_{v v^{\prime}} \delta_{j j^{\prime}} \delta_{n n^{\prime}}, \\
\hat{H}_{F}^{(k)}(\mathbf{r})=T^{-1} \int_{0}^{T} d t \exp (i k \omega t) \hat{H}(\mathbf{r}, t),
\end{gathered}
$$

and

$$
\begin{aligned}
& \left\langle v j\left|\hat{H}_{F}^{(k)}(\mathbf{r})\right| v^{\prime} j^{\prime}\right\rangle=\int_{0}^{\infty} d r \int_{-1}^{+1} d \cos \theta\left[\zeta_{v}(r) P_{j}^{m_{j}}(\cos \theta)\right]^{*} \\
& \quad \times \hat{H}_{F}^{(k)}(\mathbf{r})\left[\zeta_{v^{+}}(r) P_{j^{\prime}}^{m_{j}}(\cos \theta)\right],
\end{aligned}
$$

where $P_{f}^{m_{j}}(\cos \theta)$ is the orthonormalized Legendre polynomial. The Floquet Hamiltonian $\hat{H}_{F}$, in the Floquet state basis $\{|v j, n\rangle\}$, can be represented by a block-tridiagonal infinite Hermitian matrix (Fig. 1) with rows and columns denoted by the trio of indices $v, j, n$. In practice, the Floquet matrix is truncated to $N$ by $N$ in dimension with $N=N_{F} N_{\gamma} N_{J}$, where $N_{F}$ is the number of Floquet photon (Fourier) blocks, $N_{V}$ the number of vibrational levels included in one Floquet block, and $N_{\delta}$ the number of rotational states included in one vibrational level. Figure 1 shows that $\hat{H}_{F}$ has a periodic structure with only the number of $\omega$ 's in the diagonal elements varying from block to block. The structure endows the quasienergy eigenvalues and eigenvectors of $\hat{H}_{F}$ with periodic properties. The time evolution unitary operator $U\left(t ; t_{0}\right)$, obeying the initial condition 
$U\left(t_{0} ; t_{0}\right)=1$, in the matrix representation [in terms of the molecular basis $\left.|v j\rangle=\zeta_{v}(r) Y_{j m_{j}}(\theta, \phi)\right]$ is merely

$\left\langle v^{\prime} j^{\prime}\left|U\left(t_{;} t_{0}\right)\right| v j\right\rangle=\sum_{\epsilon_{\beta}}\left\langle v^{\prime} j^{\prime} \mid \Psi_{\epsilon_{\beta}}(\mathbf{r}, t)\right\rangle \cdot\left\langle\Psi_{\epsilon_{\beta}}\left(\mathbf{r}, t_{0}\right) \mid v j\right\rangle$,

where

$\left\langle v j \mid \Psi_{\epsilon_{\beta}}(\mathbf{r}, t)\right\rangle=\exp \left(-i \epsilon_{\beta} t\right) \sum_{n=-\infty}^{\infty} \exp (i n \omega t)\left\langle v j, n \mid \epsilon_{\beta}\right\rangle$.

\section{B. Nonadiabatic approach to multiphoton excitation quantum dynamics}

In the infrared multiphoton absorption (IR MPA) processes, the laser frequency $\omega$ is close to the characteristic frequency of the molecular vibration and considerably exceeds the rotational frequency. Analogous to the Born-Oppenheimer approximation, one can first solve the fast vibrational motion in the field with the rotational motion frozen. The resulting Schrödinger equation (with $\theta$ fixed) is

$i \partial \Phi(r, t ; \theta) / \partial t=\left[{\hat{H_{v}}}^{(0)}(r)-\mu(r) E_{0} \cos \theta \cos \omega t\right] \Phi(r, t ; \theta)$,

yielding a quasivibrational energy (QVE) which depends parametrically on the orientation of the molecule. The slow rotational motion can then be solved by using the QVE as the effective potential energy. Based on this procedure, a computationally efficient yet reliable method can be formulated.

Since the vibrational-field Hamiltonian, $\hat{H}_{v}^{(0)}(r)$ $-\mu(r) E_{0} \cos \theta \cos \omega t$, is a periodic function of time $t$ with the period $T=2 \pi / \omega$, Floquet theorem ${ }^{2,3,10}$ again assures the existence of the solutions $\Phi_{\alpha k}$ of Eq. (13) of the form ( $\alpha=$ vibrational index, $k=$ photon index)

$$
\Phi_{\alpha k}(r, t ; \theta)=\exp \left(-i \lambda_{\alpha k} t\right) f_{\alpha k}(r, t ; \theta)
$$

and

$$
f_{\alpha k}(r, t+T ; \theta)=f_{\alpha k}(r, t ; \theta),
$$

where $\lambda_{\alpha k}(\theta)$ is called the quasivibrational energy (QVE) and the functions $\left\{f_{\alpha k}\right\}$ form a complete orthonormal set and are eigenfunctions of the following eigenvalue equation:

$$
\begin{aligned}
{\left[\hat{H}_{v}^{(0)}(r)\right.} & \left.-\mu(r) E_{0} \cos \theta \cos \omega t-i \partial / \partial t\right] f_{\alpha k}(r, t ; \theta) \\
& =\lambda_{\alpha k}(\theta) f_{\alpha k}(r, t ; \theta) .
\end{aligned}
$$

Analogous to Eq. (5), Eq. (16) can be transformed into a static problem by expanding $f_{\alpha k}$ into a Fourier series

$$
f_{\alpha k}(r, t ; \theta)=\sum_{n=-\infty}^{\infty} \exp (i n \omega t) \sum_{\nu}\left\langle v, n \mid \lambda_{\alpha k}\right\rangle_{\theta} \zeta_{\nu}(r) .
$$

The expansion coefficients $\left\langle v, n \mid \lambda_{\alpha k}\right\rangle_{\theta}$ in Eq. (17) can be determined from the solution of the Floquet matrix

\begin{tabular}{|c|c|c|c|}
\hline Where & $v=0$ & $v=1$ & $v=2$ \\
\hline \multirow{3}{*}[A]{$=$} & $\mathrm{E}_{0}^{(0)}$ & 0 & 0 \\
\hline & 0 & $E_{1}^{(0)}$ & 0 \\
\hline & 0 & 0 & $E_{2}^{(0)}$ \\
\hline and & $v=0$ & $v=1$ & $v=2$ \\
\hline \multirow{3}{*}[B(\theta)]{$=$} & $a_{00}(\theta)$ & $a_{0 s}(\theta)$ & $a_{02}(\theta)$ \\
\hline & & $a_{11}(\theta)$ & $a_{12}(\theta)$ \\
\hline & & & $a_{22}(\theta)$ \\
\hline
\end{tabular}
eigenvalue equation

$\sum_{v^{\prime}} \sum_{n^{\prime}}\left\langle v, n\left|\hat{H}_{F}^{(v)}(\theta)\right| v^{\prime} n^{\prime}\right\rangle\left\langle v^{\prime} n^{\prime} \mid \lambda_{\alpha k}\right\rangle_{\theta}=\lambda_{\alpha k}(\theta)\left\langle v n \mid \lambda_{\alpha k}\right\rangle_{\theta}$,

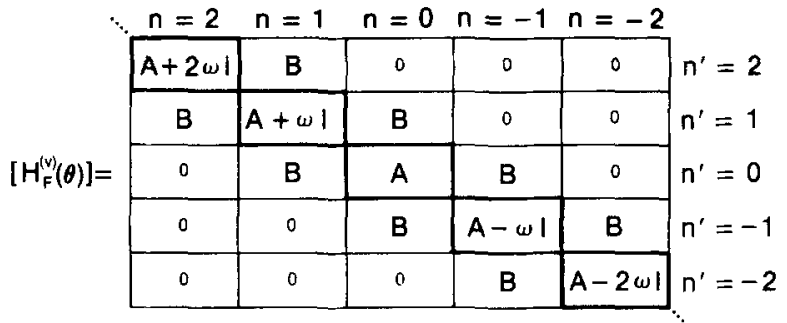

FIG. 2. Structure of the orientation $(\theta)$-dependent vibrationalfield Floquet Hamiltonian $\hat{H}_{F}^{(v)}(\theta)$ in the Floquet state basis $\{|v, n\rangle\} . \quad E_{v}^{(0)}$ are the unperturbed vibrational energies, and $a_{v v}(\theta)$ are electric dipole coupling matrix elements. For further explanation see the caption of Fig. 1.

where

$\left\langle v, n\left|\hat{H}_{F}^{(v)}(\theta)\right| v^{\prime} n^{\prime}\right\rangle=\left\langle v\left|\hat{H}_{v F}^{\left(n-n^{\prime}\right)}(r, \theta)\right| v^{\prime}\right\rangle+n \omega \delta_{v v^{\prime}} \delta_{n n^{\prime}}$,

$\hat{H}_{v F}^{(n)}(r, \theta)=T^{-1} \int_{0}^{T} d t\left[H_{v}^{(0)}(r)\right.$

$$
\left.-\mu(r) E_{0} \cos \theta \cos \omega t\right] \cdot \exp (i n \omega t),
$$

and

$$
\left\langle v\left|\hat{H}_{v F}^{(n)}(r, \theta)\right| v^{\prime}\right\rangle=\int_{0}^{\infty} d r \zeta_{v}(r) \hat{H}_{v F}^{(n)}(r, \theta) \zeta_{v^{\prime}}(r) .
$$

The resulting Floquet Hamiltonian $\hat{H}_{F}^{(v)}(\theta)$, in the Floquet (or vibrational-field) state basis $\{|v, n\rangle\}$, can be represented by a block-tridiagonal infinite Hermitian matrix as depicted in Fig. 2. In practice, the Floquet matrix is truncated by $N$ by $N$ in dimension with $N=N_{F} N_{v}$, where $N_{F}$ is the number of Floquet photon (Fourier) blocks and $N_{\nu}$ the number of vibrational levels included in one Floquet block. Similar to the exact Floquet Hamiltonian $\hat{H}_{F}$ (Fig. 1), the vibrational Floquet Hamiltonian $\hat{H}_{F}^{(v)}(\theta)$ also possesses a periodic structure with only the number of $\omega^{\prime} s$ in the diagonal elements varying from block to block. This endows the QVE's and their eigenfunctions with periodic properties.

With the assumption that the inf rared laser frequency $\omega$ is close to the energy difference of adjacent vibrational states of interest, one finds that the QVE spec$\operatorname{trum}\left\{\lambda_{\alpha k}(\theta)\right\}$ possesses a well-defined band structure characterized by the frequency $\omega$. By rewriting the subscripts $(\alpha k)$ as $(\alpha,-\alpha+P)$, with $P$ an arbitrary integer, the bands are then indexed by $P$ and the sublevels within a given $P$ band are ordered according to $\alpha(\alpha=0,1,2 \ldots)$. Each intraband sublevel is assigned a 
QVE $\lambda_{\alpha,-\alpha+P}(\theta)$ and a wave function $f_{\alpha,-\alpha+P}(r, t ; \theta)$, which have the following periodic properties:

$$
\lambda_{\alpha,-\alpha+P}(\theta)=\lambda_{\alpha,-\alpha}(\theta)+P \omega=\lambda_{\alpha, 0}(\theta)+(P-\alpha) \omega
$$

and

$$
f_{\alpha,-\alpha+P}(r, t ; \theta)=f_{\alpha,-\alpha}(r, t ; \theta) \cdot \exp (i P \omega t) .
$$

Equations (22) suggest that all the $P$ bands possess the same QVE structure since

$$
\begin{aligned}
\Phi_{\alpha,-\alpha+P_{1}}(r, t ; \theta)=\Phi_{\alpha,-\alpha+P_{2}}(r, t ; \theta), \quad P_{1} & \neq P_{2}, \\
\alpha & =0,1,2, \ldots .
\end{aligned}
$$

The QVE's within each $P$ band, consisting of the subset $\left\{\lambda_{\alpha,-\alpha+p}\right\}$, are congregations of the QVE levels which are nearly degenerate and strongly coupled via slow rotational motion in the sequential IR MPA process

$|v=0, N+P\rangle \stackrel{\omega}{\rightarrow}|v=1, N+P-1\rangle \stackrel{\omega}{\longrightarrow}|v=2, N+P-2\rangle \stackrel{\omega}{\rightarrow}$ ect.

where $N$ is a very large integer characteristic of the number of photons present in the physical absorption process, and $P$ indexes the QVE band. It is thus apparent that all QVE $P$ bands portray the same physical process, viz. the sequential photoabsorption. The intraband width is determined by the number of vibrational states $\left(N_{v}\right)$ of interest, and is in general much smaller than the interband separation $\omega$. As a result, the couplings between the interband QVE levels due to the slow rotation of the molecule are much weaker than that between the intraband ones. The relationship of each QVE $P$ band to the molecular rotation is analogous to that of a molecular electronic state to the molecular nuclear motion in the framework of Born-Oppenheimer approximation. Thus to an excellent approximation, the IR MPA processes of interest can be properly studied within any $P$ band to be described below.

In the spirit of the Born-Oppenheimer approximation, the total quasi-vibrational-rotational states (indexed by $\beta$ ) are described by wave functions of the form

$$
\begin{aligned}
\Psi_{\epsilon_{\beta}}^{(P)}(\mathbf{r}, t)= & \exp \left(-i \epsilon_{\beta} t\right)(2 \pi)^{-1 / 2} \exp \left(i m_{j} \phi\right) \\
& \times \sum_{\alpha} \chi_{\beta \alpha}^{(P)}(\theta) f_{\alpha_{1}-\alpha_{+} P}(r, t ; \theta),
\end{aligned}
$$

where $\alpha$ runs over all QVE's in the $P$ band, and $\chi_{\beta \alpha}^{(P)}$ is the wave function describing the rotational motion of the diatom under the potential provided by the quasivibrational energies within the $P$ band, calculated as if the rotation of the molecule were frozen. For an arbitrary quasi-vibrational-rotational state of a fixed total quasienergy $\epsilon_{B}$, the exact total quasienergy state wave function may be expanded as

$$
\Psi_{\epsilon_{B}}(\mathbf{r}, t)=\sum_{P} \Psi_{\epsilon_{B}}^{(P)}(\mathbf{r}, t) .
$$

Again, we have assumed that the system embodies a well-defined quantum number $m_{j}$. Substituting Eq. (24) into Eq. (1), and projecting onto any other $f_{\alpha^{\prime},-\alpha^{c}+P^{\prime}}(r, t ; \theta)$ state (i.e., integration over a period of the field oscillation is carried out along with the $r$ integration) lead to the equation for the rotational motion of the molecule

$$
\begin{aligned}
& \hat{H}_{\text {rot }}(\theta) \chi_{\beta \alpha}(\theta)+\left(U_{\alpha m_{j}}^{(P)}-\epsilon_{\beta}\right) \chi_{\beta \alpha}^{(P)}(\theta) \\
& +\sum_{\alpha^{\prime \neq \alpha}} V_{\alpha \alpha^{\prime}}^{\left(P P^{\prime}\right)} \chi_{\beta \alpha^{\prime}}^{(P)}(\theta)+\sum_{P^{\prime} \neq P} \sum_{\alpha^{\prime}} V_{\alpha \alpha^{\prime}}^{\left(P P^{\prime}\right)} \chi_{\beta \alpha^{\prime}}^{\left(P^{\prime}\right)}(\theta),
\end{aligned}
$$

where

$$
\begin{aligned}
\hat{H}_{\text {rot }}= & -B_{\theta}(\sin \theta)^{-1}(\partial / \partial \theta)(\sin \theta \partial / \partial \theta), \\
U_{\alpha m_{j}}^{(P)}= & \lambda_{\alpha,-\alpha+P}(\theta)+B_{\theta} m_{j}^{2}(\sin \theta)^{-2}+y_{\alpha \alpha}^{(P P)}(\theta), \\
V_{\alpha \alpha^{\prime}}^{\left(P P^{\prime}\right)}= & y_{\alpha \alpha^{\prime}}^{\left(P P^{\prime}\right)}(\theta)-2 B_{\theta} \sum_{\nu} \sum_{n}\left\langle v n \mid \lambda_{\alpha,-\alpha+P}\right\rangle_{\theta} \\
& \times\left(\frac{\partial}{\partial \theta}\left\langle v n \mid \lambda_{\alpha^{\prime},-\alpha^{\prime}+P^{\prime}}\right\rangle_{\theta} \frac{\partial}{\partial \theta}\right),
\end{aligned}
$$

and

$y_{\alpha \alpha^{\prime}}^{\left(P P^{\prime \prime}\right)}(\theta)=\sum_{v} \sum_{n}\left\langle v n \mid \lambda_{\alpha,-\alpha+P}\right\rangle_{\theta} \hat{H}_{\mathrm{rot}}\left\langle v n \mid \lambda_{\alpha^{\prime},-\alpha^{\prime}+P^{\prime}}\right\rangle_{\theta}$.

Due to the large separation between the QVE bands and the slow variation of the quantity $\left\langle v n \mid \lambda_{\alpha,-\alpha+P}\right\rangle_{\theta}$ with $\theta$, the interband rotational couplings $V_{\alpha \alpha^{\prime}}^{\left(P P^{\prime j}\right)}\left(P \neq P^{\prime}\right)$ are orders of magnitude smaller than those of intraband $\left(P=P^{\prime}\right)$. Thus the second summation $\left(\sum_{P^{\prime} \neq P}\right)$ in the right-hand side of Eq. (25) may be safely ignored, yielding the equation for the rotational motion in the $P$ band

$\hat{H}_{\text {rot }}(\theta) \chi_{\beta \alpha}^{(P)}(\theta)+\left(U_{\alpha m_{j}}^{(P)}-\epsilon_{\beta}\right) \chi_{\beta \alpha}^{(P)}(\theta)=\sum_{\alpha^{\prime} \neq \alpha} V_{\alpha \alpha^{\prime}}^{\left(P P^{\prime}\right)} \chi_{\beta \alpha}^{(P)}(\theta)$.

Since all the $P$ bands possess the same QVE structure to within a phase factor, only one $P$ band needs to be considered. For convenience we shall choose the $P=0$ band below and the index $P$ will be dropped.

The nonadiabatic [in the sense that we still keep the intraband off-diagonal coupling terms $\left.V_{\alpha \alpha^{*}}\left(\alpha \neq \alpha^{\prime}\right)\right]$ coupled equations in Eq. (30) can be solved most expediently by expanding the wave function $\chi_{\beta \alpha}(\theta)$ in terms of orthonormalized associated Legendre polynomials $P_{j}^{m}(\cos \theta)$,

$$
\chi_{\beta \alpha}(\theta)=\sum_{j=\left|m_{j}\right|} b_{\beta, \alpha j} P_{\xi}^{m_{j}}(\cos \theta) .
$$

Substituting Eq. (31) into Eq. (30), we obtain the following eigenvalue equation:

$$
\sum_{\alpha^{\prime}} \sum_{j^{\prime}} g_{\alpha \alpha^{\prime}}^{j j^{\prime \prime}} b_{\beta, \alpha^{\prime} j^{\prime}}=\epsilon_{\beta} b_{\beta, \alpha J}
$$

where

$$
\begin{aligned}
g_{\alpha \alpha^{\prime}}^{j j^{\prime}=} & \delta_{\alpha \alpha^{\prime}} \cdot \int_{-1}^{1} d \cos \theta P_{j}^{m_{j}}(\cos \theta) \lambda_{\alpha}(\theta) P_{j^{\prime}}^{m_{j}}(\cos \theta) \\
& +\sum_{v} \sum_{n} \int_{-1}^{1} d \cos \theta\left[P_{j}^{m_{j}}(\cos \theta) F_{v n_{,} \alpha}(\theta)\right] \\
& \times \hat{H}_{R R}(\theta, \phi)\left[F_{v n_{,} \alpha^{\prime}}(\theta) P_{j^{\prime}}^{m_{j}}(\cos \theta)\right] .
\end{aligned}
$$

Here we have adopted new notations: $\lambda_{\alpha}(\theta)$ for $\lambda_{\alpha,-\alpha}(\theta)$ and $F_{v n_{,} \alpha}(\theta)$ for $\left\langle v n \mid \lambda_{\alpha,-\alpha}\right\rangle_{\theta}$. The structure of the total quasienergy “ $G$ " Hamiltonian, ${ }^{11}$ namely $\left\{g_{\alpha \alpha}^{j j} \cdot\right\}$, is depicted in the lower portion of Fig. 3. The upper portion in Fig. 3 is what Eq. (32) would have been, had we employed Eq. (25).

With the help of Eqs. (17), (23), and (31), the set of the total quasi-vibrational-rotational state wave func- 


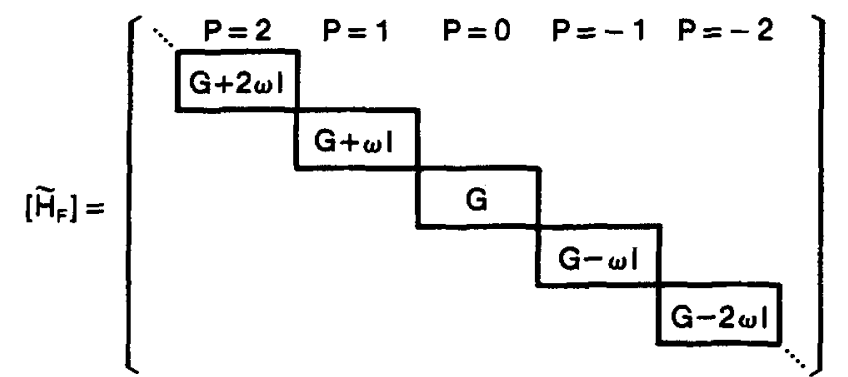

\begin{tabular}{|c|c|c|c|}
\hline Where & $v=0$ & $v=1$ & $v=2$ \\
\hline \multirow{3}{*}[\mathrm{G}]{$=$} & $\left(G_{00}\right)$ & $\left(G_{01}\right)$ & $\left(G_{02}\right)$ \\
\hline & & $\left(G_{11}\right)-\omega \mid$ & $\left(G_{12}\right)$ \\
\hline & & & $\left|\left(G_{22}\right)-2 \omega\right|$ \\
\hline and & $j=0$ & $j=1$ & $j=2$ \\
\hline \multirow{3}{*}{$\left(G_{w^{\prime}}\right)$} & $g_{v v^{\prime}}^{\infty}$ & $g_{w}^{01}$ & $g_{w}^{02}$ \\
\hline & & $g_{v v^{\prime}}^{11}$ & $g_{v v^{\prime}}^{12}$ \\
\hline & & & $g_{v v^{\prime}}^{22}$ \\
\hline
\end{tabular}

FIG. 3. The approximate band structure (indexed by $P$ ) of the quasivibrational energy ( $Q V E)$ spectrum is shown in the topmost diagram. Only the $P=0$ band (i.e., the central $G$ block) needs to be considered in the total quasienergy calculations. Shown here is also the structure of the $G$ matrix in the QVE and rotational basis.

tions obtained from the nonadiabatic method become

$$
\begin{aligned}
& \Psi_{\epsilon_{\beta}}(\mathrm{r}, t)=\exp \left(-i \epsilon_{\beta} t\right) \cdot(2 \pi)^{-1 / 2} \exp \left(i m_{j} \phi\right) \\
& \quad \times \sum_{n} \sum_{\alpha} \sum_{\nu} \sum_{j} \exp (i n \omega t) b_{\beta, \alpha j} F_{m_{,} \alpha}(\theta) \cdot \zeta_{v}(r) P_{j}^{m_{j}}(\cos \theta) .
\end{aligned}
$$

By combining Eqs. (11) and (34), and after some manipulations, the time evolution operator $U\left(t ; t_{0}\right)$ can be written, in the matrix form, explicitly as

$$
\begin{aligned}
& \left\langle v^{\prime} j^{\prime}\left|U\left(t ; t_{0}\right)\right| v j\right\rangle=\sum_{n} \sum_{\epsilon_{B}}\left\{\left\langle v^{\prime} j, n \mid \epsilon_{B}\right\rangle\right. \\
& \left.\quad \times \exp \left[-i \epsilon_{B}\left(t-t_{0}\right)\right] \cdot\left\langle\epsilon_{B} \mid v j, 0\right\rangle \cdot \exp (i n \omega t)\right\},
\end{aligned}
$$

where $e^{12}$

$$
\begin{aligned}
\left\langle v j, n \mid \epsilon_{B}\right\rangle \equiv & \sum_{\alpha^{\prime}} \sum_{j^{\prime}} b_{\beta, \alpha^{\prime} j^{\prime}} \int_{-1}^{+1} d \cos \theta \\
& \times P_{j}^{m_{j}}(\cos \theta) \cdot F_{v n, \alpha^{\prime}}(\theta) \cdot P_{j^{\prime}}^{m_{j}}(\cos \theta) .
\end{aligned}
$$

Transition probability going from the initial vibrationalrotational state $\left|v j m_{j}\right\rangle$ (with $m_{j}$ staying constant in time) to the final state $\left|v^{\prime} j^{\prime} m_{j}\right\rangle$ is given as

$$
P_{v j-v^{\prime} j^{\prime}}^{\left(m_{j}\right)}\left(t, t_{0}\right)=\left|\left\langle v^{\prime} j^{\prime}\left|U\left(t ; t_{0}\right)\right| v j\right\rangle\right|^{2} .
$$

Of the most experimental interests, averaging Eq. (37) over the initial time $t_{0}$, while keeping the elapsed time $\left(t-t_{0}\right)$ fixed, yields

$$
\begin{aligned}
P_{v j=v^{\prime} j^{\prime}}^{\left(m_{j}\right)}\left(t-t_{0}\right)= & \sum_{n} \mid \sum_{\epsilon_{\beta}}\left\langle v^{\prime} j^{\prime}, n \mid \epsilon_{\beta}\right\rangle \\
& \times\left.\exp \left[-i \epsilon_{\beta}\left(t-t_{0}\right)\right] \cdot\left\langle\epsilon_{\beta} \mid v j, 0\right\rangle\right|^{2} .
\end{aligned}
$$

Finally, further averaging Eq. (38) over $\left(t-t_{0}\right)$ results in the long-time average transition probability

$$
\bar{P}_{v j^{\prime} v^{\prime} j^{\prime}}^{\left(m_{j}^{\prime}\right.}=\sum_{n} \sum_{\epsilon_{B}}\left|\left\langle v^{\prime} j^{\prime}, n \mid \epsilon_{\beta}\right\rangle \cdot\left\langle\epsilon_{\beta} \mid v j, 0\right\rangle\right|^{2} .
$$

\section{RESULTS AND DISCUSSIONS}

The nonadiabatic approach outlined in Sec. II B has been used to study the quantum dynamics of the multiphoton excitation of the ${ }^{12} \mathrm{C}^{16} \mathrm{O}$ molecule in the presence of the strong infrared laser fields. We have assumed that the $\mathrm{CO}$ molecule can be represented by a rotating Morse oscillator with the Morse Hamiltonian given by

$$
\hat{H}_{v}^{(0)}(r)=-(2 M)^{-1} d^{2} / d r^{2}+D_{e}\left\{1-\exp \left[-\alpha\left(r-r_{e}\right)\right]\right\}^{2} .
$$

The molecular electric dipole moment adopted in the calculation has the linear form $\mu(r)=\mu_{0}+\mu_{1}\left(r-r_{\theta}\right)$ which is adequate for MPE of the low-lying vibrational states. Throughout the calculation, the field strength of the IR laser is assumed to be $50 \mathrm{GW} / \mathrm{cm}^{2}$, and the MPA analysis is made for the vibrational states up to $v=3$ covering field frequencies ranging from 2115 to $2165 \mathrm{~cm}^{-1}$. The choice of the frequency range will become evident in the results. The molecular parameters. ${ }^{13}$ used for $\mathrm{CO}$ are tabulated in Table I.

\section{A. QVE spectrum and wave functions}

In calculating the QVE spectrum $\left\{\lambda_{\alpha, \alpha+P}(\theta)\right\}$ and the corresponding wave functions $\left\{f_{\alpha,-\alpha+P}(r, t ; \theta)\right\}$, we have solved the eigenvalue Eq. (18). The vibrational Floquet Hamiltonian matrix (Fig. 2) was truncated to contain only five vibrational levels (i.e., $v=0,1,2,3,4$ ) and nine photon blocks (i. e., $n=0, \pm 1, \pm 2, \pm 3$, and \pm 4 ), but enough to achieve convergences of the one- , two-, and three-photon transitions which are of our current concern. Figures $4(a)$ and $4(b)$ depict the representative results of the QVE spectrum $\left\{\lambda_{\alpha}(\theta)\right\}$ and various components of the corresponding eigenvector $F_{v n, \alpha}(\theta)$ of the $P=0$ band at the field frequency $\omega=2131.7 \mathrm{~cm}^{-1}$ nearby the resonance (i.e., $v=0 \leftrightarrow v=2$ ) region. The appealing symmetry of $\lambda_{\alpha}(\theta)$ and $F_{v n, \alpha}(\theta)$ about the plane perpendicular (i.e., $\theta=90^{\circ}$ ) to the direction of the field can be attributed to the existence of a unitary transformation matrix ${ }^{14}$ linking the two block tridiagonal matrices $H_{F}^{(\nu)}(\theta)$ and $H_{F}^{(\nu)}(\pi-\theta)$ :

TABLE I. ${ }^{12} \mathrm{C}^{16} \mathrm{O}$ molecular parameters. $^{2}$

$\begin{aligned} D_{\theta} & =10.982 \mathrm{eV} \\ \alpha & =1.2302 a_{0}^{-1} \\ r_{B} & =2.132 a_{0} \\ M & =6.856182283 \mathrm{amu} \\ B_{\theta} & =1.931 \mathrm{~cm}^{-1} \\ \omega_{\theta} & =2169.81 \mathrm{~cm}^{-1} \\ x_{8} \omega_{B} & =13.288 \mathrm{~cm}^{-1} \\ \mu_{0} & =0.112 \mathrm{D} \\ \mu_{1} & =1.598 \mathrm{D} / a_{0}\end{aligned}$

${ }^{2}$ Reference 13. 

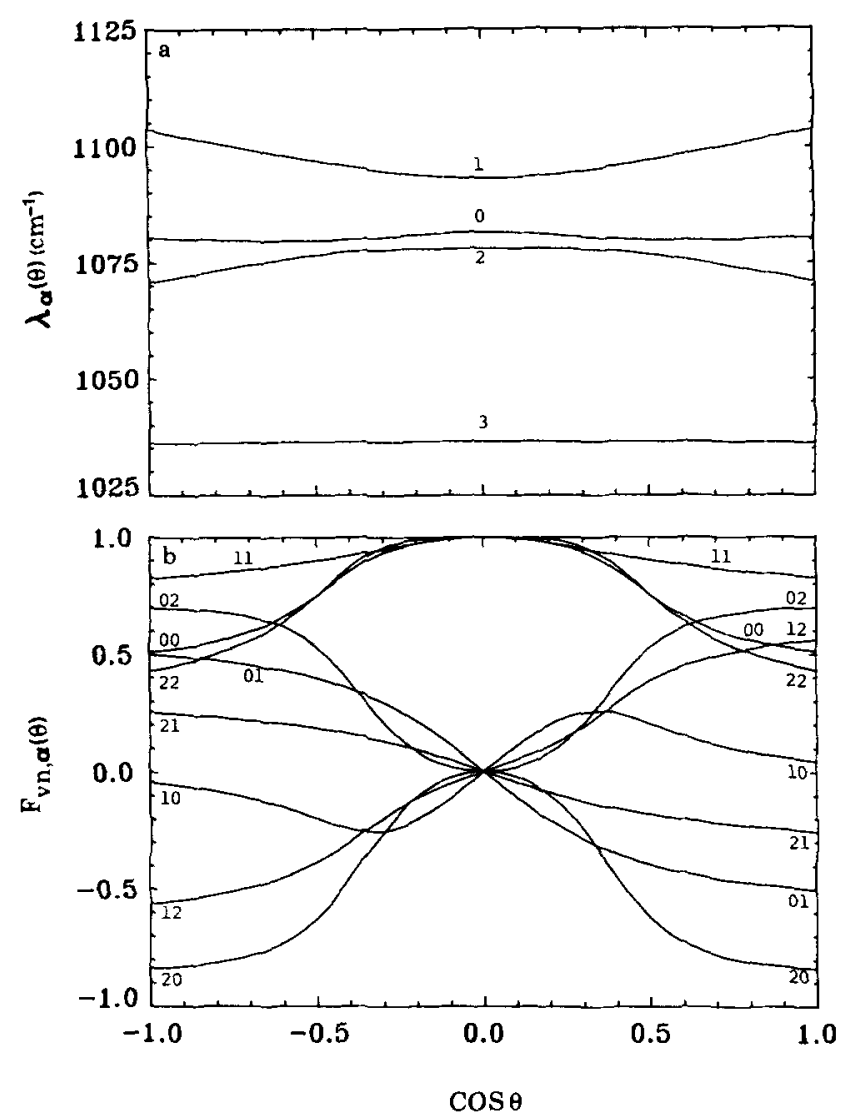

FIG. 4. (a) Orientation-dependent quasivibrational energies $\lambda_{\alpha}(\theta)(\alpha=0,1,2,3)$ and $(b)$ their corresponding representative dominant eigenfunction components (with $n=-v$ ) of $F_{\text {in, } \alpha}(\theta)$ (denoted by $v \alpha$ in the figure) for ${ }^{12} \mathrm{C}^{16} \mathrm{O}$ at $50 \mathrm{GW} / \mathrm{cm}^{2}$ and $\omega=2131.70 \mathrm{~cm}^{-1}$.

$$
H_{F}^{(v)}(\pi-\theta)=Q^{+} H_{F}^{(v)}(\theta) Q,
$$

where

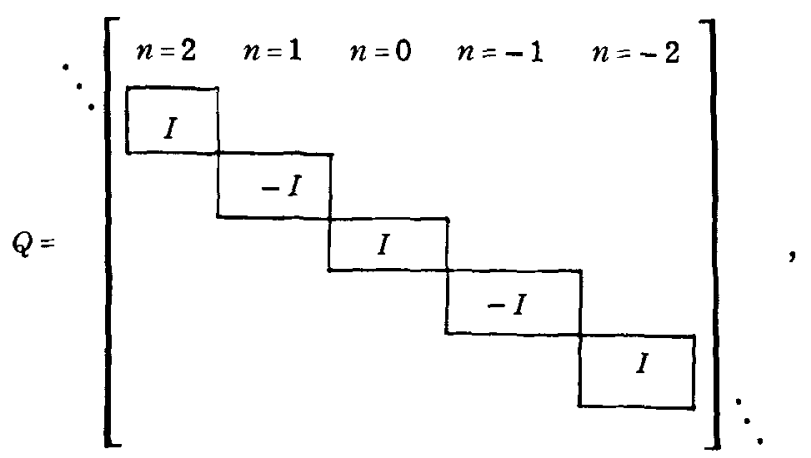

with $I$ being the indentity matrix of the dimension the same as any Floquet photon block in Fig. 2. One can easily find that the matrix $Q$ is not only unitary but also Hermitian. The symmetry properties of both $\lambda_{\alpha}(\theta)$ and $F_{v n, \alpha}(\theta)$ can be best represented by relations

$$
\lambda_{\alpha}(\pi-\theta)=\lambda_{\alpha}(\theta)
$$

and

$$
F_{v n, \alpha}(\pi-\theta)=(-1)^{n+\alpha} F_{v n_{,} \alpha}(\theta),
$$

which reveal the nature of the interaction of the field with the diatomic molecule, i. e., the $\cos \theta$ dependence, and, therefore, preserve the correct dipole selection rules on the multiphoton vibrational-rotational transitions of the molecule in the presence of the laser field. Furthermore, we noticed that the quantities $\lambda_{\alpha}(\theta)$ and $F_{v n_{0} \alpha}(\theta)$ are both slowly varying functions of the polar angle $\theta$, suggesting that only a few (typically $8 \sim 10$ ) molecular orientations are required for accurate description of the QVE's.

\section{B. Effective adiabatic vibrational potentials and nonadiabatic coupling terms}

The relatively slow rotation of the molecule is governed by Eq. (30), assuming that the rotational transition is confined within one specific QVE (say $P=0$ ) band. This is fully justified in the case of $\mathrm{CO}$. Figures $5(a)-5(d)$ show typical examples of the orientation $(\theta)$ dependent effective adiabatic vibrational potentials $U_{\alpha m_{j}}(\theta)$ [defined in Eq. (27)] for $m_{j}=0,1,2$, and 3, respectively, corresponding to $\omega=2131.70 \mathrm{~cm}^{-1}$. The adiabatic potential curves exhibit a strong dependence on the quantum number $m_{j}$ because of the factor $B_{e} m_{j}^{2} /$ $\sin ^{2} \theta$ in Eq. (27). For $m_{j}=0$, the molecule is allowed to swing through all angles. However, for the case of $m_{j} \neq 0$, the potential becomes very steep nearby the field directions (namely $\theta=0^{\circ}$ and $180^{\circ}$ ) and the rotational motion is confined to a more limited space in the $\theta$ configuration. The larger the quantum number $m_{f}$, the more restriction the rotational motion will be. The features embedded in these adiabatic potentials are exhibited in the MPA spectrum to be discussed later.

The nonadiabatic coupling terms $V_{\alpha \alpha}$ (Eq. 30) are the main driving mechanisms for inducing intraband vibrational-rotational transitions in MPA processes. These coupling terms become crucially important nearby resonant transition regions where the quasivibrational energies are nearly degenerate. As an example, Figs. $6(\mathrm{a})$ to $6(\mathrm{c})$ depict the primary coupling terms $y_{\alpha \alpha^{\prime}}(\theta)$ [Eqs. (28) and (29)] as a function of $\theta$ for the three strongly coupled and nearly degenerate quasivibrational levels $\left(\alpha, \alpha^{\prime}=0,1,2\right)$ for the case of $\omega=2131.70 \mathrm{~cm}^{-1}$. Notice that the magnitude of $y_{\alpha \alpha^{\prime}}\left(\alpha \neq \alpha^{\prime}\right)$ are comparable with or even larger than the quasivibrational level spacings, indicating the importance of these intraband coupling terms in determining the proper vibrational-rotational transitions. Also shown here are the diagonal terms $y_{\alpha \alpha}$, which are responsible for the slowly wiggly structure of the adiabatic potential curves in Figs. $5(a)-5(d)$.

\section{Comparison with the exact Floquet calculation}

Figures $7(a)$ and $7(b)$ show, respectively, the final converged results, obtained by the nonadiabatic approximation method, for the total quasi-vibrational-rotational energies $\epsilon_{\beta}^{(m j m 0)}$ (to within $0.001 \mathrm{~cm}^{-1}$ ) and the time average transition probabilities $\bar{P}_{00-v j}$ [Eq. (39)] as functions of the laser frequency $\omega$ for the MPA process $\operatorname{CO}\left(v=j=m_{j}=0\right)+n \omega-\operatorname{CO}\left(v^{\prime} j^{\prime}, m_{j}=0\right)$. The vibrational Floquet Hamiltonian matrix $H_{F}^{(u)}(\theta)$ is trancated to contain nine Floquet photon blocks (i.e., $n=0$, $\pm 1, \pm 2, \pm 3, \pm 4$ in Fig. 2), and five vibrational levels 


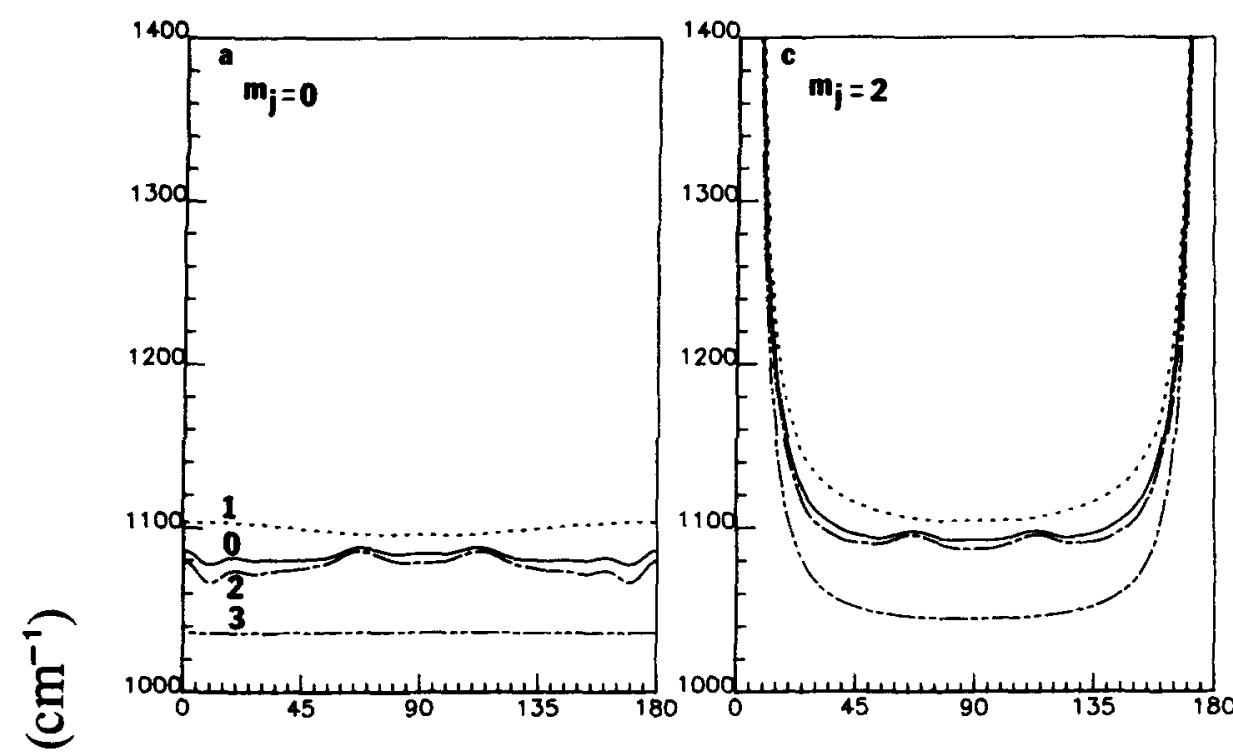

FIG. 5. Orientation-dependent effective adiabatic vibrational potentials $U_{\alpha m j}(\theta)$ for (a) $m_{j}=0$, (b) $m_{j}=1$, (c) $m_{j}=2$, and (d) $m_{j}=3$ for ${ }^{12} \mathrm{C}^{16} \mathrm{O}$ at $50 \mathrm{GW} / \mathrm{cm}^{2}$ and $\omega=2131.70 \mathrm{~cm}^{-1}$.

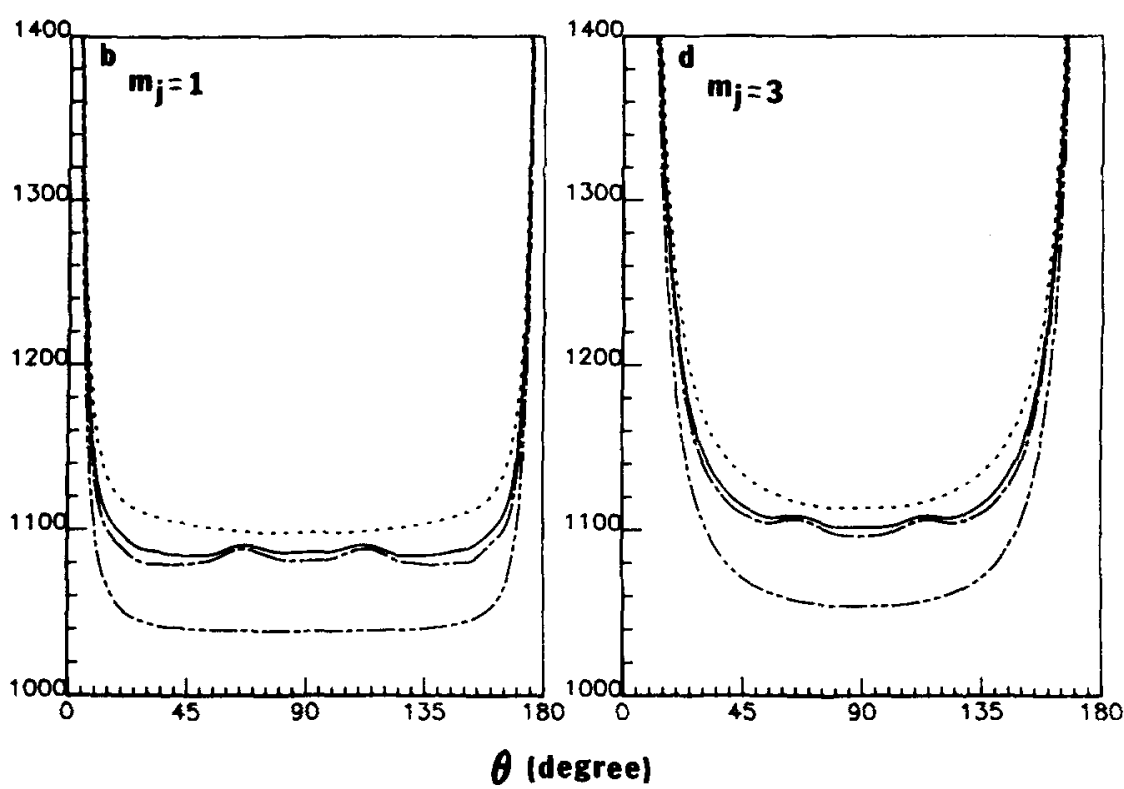

Curve notations are - for $\alpha=0$, -.- for $\alpha=1, \ldots$ for $\alpha=2$, and -

$(v=0,1,2,3,4)$. The nonadiabatic $G$-matrix analysis (Fig. 3) was carried out with $N_{V}=5$ and $N_{J}=8$ (i.e., $j=0,1,2, \ldots 7)$. Notice that the matrix elements $g_{\alpha \alpha}^{j,}$, of the $G$ matrix satisfy the important symmetry relation

$$
g_{\alpha \alpha^{\prime}}^{j j^{\prime}} \equiv 0, \text { if }\left(\alpha+\alpha^{\prime}+j+j^{\prime}\right)=\text { odd integer. }
$$

This implicitly ensures that the electric dipole selection rules are appropriately installed in the nonadiabatic approach. The correlation between the quasienergy level (avoided) crossings and nonlinear MPA line shape features (such as power broadening, hole burning, Autler-Townes multiplet splitting, $S$ hump, etc.) has been studied in great detail previously. ${ }^{5}$ Shown in Fig. $7(\mathrm{c})$ is the corresponding data from the exact Floquet analysis (Fig. 1) with $N_{F}=9, N_{Y}=5$, and $N_{J}=8$. The excellent agreement of the MPA spectra in all the fine details demonstrate the reliability of this newly introduced nonadiabatic approach. In the current example, the nonadiabatic calculation is about an order of magnitude faster computationally than the exact Floquet analysis. Clearly the former method becomes even more advantageous as the dimensionality of the Floquet matrix $N\left(=N_{F} N_{V} N_{J}\right)$ grows. (This is the case when multiphoton processes of much higher order are involved and/or heavier or larger molecules, i. e., $N_{V} N_{J}$ much larger, are under investigation.) Another major advantage (and computational saving) of the nonadiabatic approach is that the orientation-dependent QVE calculations are independent of $m_{j}$. In contrast, the exact Floquet approach requires the repetition of the entire effort for each change in $m_{j}$.

\section{Multiphoton absorption spectrum}

In the previous subsection we have presented the longtime average transition probabilities of the molecule $\mathrm{CO}$, initially at the state $\left|v=j=m_{j}=0\right\rangle$, to various excited states (up to $v=3$ ) of this approach along with the exact Floquet analysis. In reality, the molecules are thermally distributed over different quantum states. Under most of the experimental environments, i.e., at or below room temperature, molecules are populated only among the rotational states of the ground vibra- 

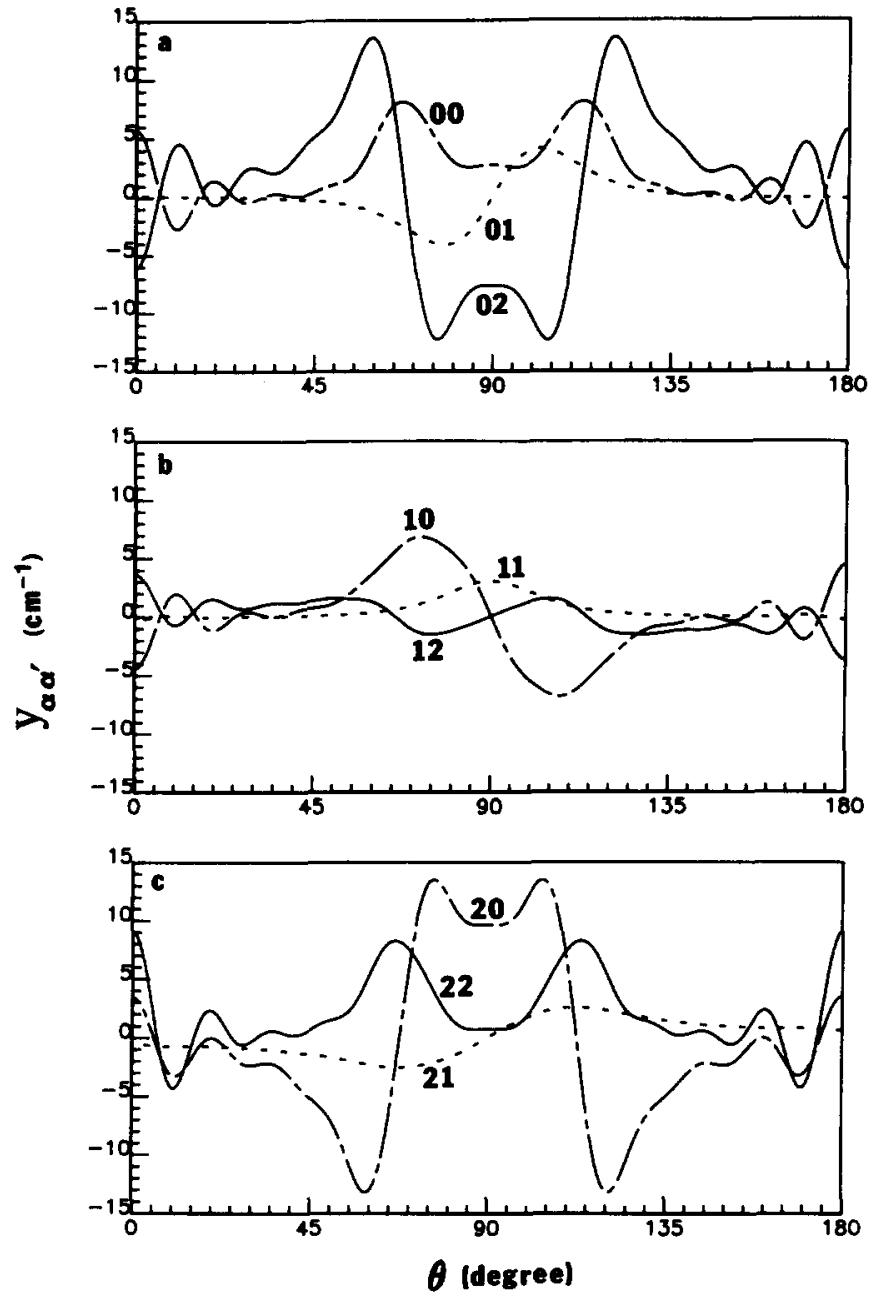

FIG. 6. Primary nonadiabatic coupling terms $y_{\alpha \alpha^{\prime}}(\theta)$ for (a) $\alpha=0$, (b) $\alpha=1$, and (c) $\alpha=2$ for ${ }^{12} \mathrm{C}^{16} \mathrm{O}$ at $50 \mathrm{GW} / \mathrm{cm}^{2}$ and $\omega=2131.70 \mathrm{~cm}^{-1}$. Curve notations are - - - for $\alpha^{\prime}=0$, $-\cdots$ for $\alpha^{\prime}=1$, and $\longrightarrow$ for $\alpha^{\prime}=2$.

tional state (i.e., $v=0$ ), and studies of the dynamics of the transitions evolved from various $v=0$ and $j$ states are essential to many spectroscopic information. In this section, we shall discuss long time average transition probabilities for (i) the molecules initially prepared at a specific $\left|v=0, j, m_{j}\right\rangle$ state, i. e., Eq. (39), (ii) the molecules initially at a state only with $v(=0)$ and $j$ specified, i. e. ,

$$
\bar{P}_{0 j \rightarrow v^{\circ} j^{*}}=\frac{1}{2 j+1} \sum_{m j^{\prime}=j}^{f} \bar{P}_{0 j \rightarrow v^{\prime} j^{\prime}}^{\left(m_{j}\right)},
$$

and finally (iii) the molecules initially thermally distributed over various $j$ states of the $v=0$ level at a certain temperature $T$, i.e.,

$$
\bar{P}_{0-v^{\prime}}(T)=\sum_{j} \sum_{j} B_{j}(T) \bar{P}_{0 j-v^{\prime} j^{\prime}},
$$

where $B_{f}$ is the rotational distribution function

$$
B_{j}(T)=(2 j+1) Q_{r}^{-1} \exp \left[-B_{a} j(j+1) h c / k T\right],
$$

with $Q_{r}$ the associated rotational partition function, $h$ the Planck constant, $c$ the velocity of light, and $k$ the
Boltzmann constant. Furthermore, we have chosen to present results of Eq. (47) for temperature $T=10$ and $50 \mathrm{~K}$ for the reason that only a limited number of the rotational states, i. e., the first five states $(j=0,1,2$, $3,4)$ of the former case and ten states $(j=0-9)$ of the latter case, are significantly populated initially.

Note that in the nonadiabatic approach, the orientation-dependent QVE calculations, being independent of $j$ and $m_{f}$, need to be carried out only once for each laser frequency $\omega$. The $G$-matrix analysis, Eq. (32), however, depends upon the magnetic quantum number $m_{j}$. In solving Eq. (32), we have expanded the rotational wave function $\chi_{\beta \alpha}(\theta)$, Eq. (31), in terms of $\left(14-\left|m_{1}\right|\right)$ associated Legendre polynomials (the highest order of polynomials is fixed at $j=14$ for different $\left|m_{j}\right| \leq 9$ ). In other words, the $G$ matrix (Fig. 3) is a truncated matrix of five vibrational blocks and $\left(14-\left|m_{f}\right|\right)$ rotational states in each vibrational block, i. e., a $\left[5 \times\left(14-\left|m_{f}\right|\right)\right] \times\left[5 \times\left(14-\left|m_{j}\right|\right)\right]$ matrix. [In actual computation, the $G$ matrix can be further reduced to two sub- $G$ matrices with smaller dimensions if use is made of the symmetry relation Eq. (45).]
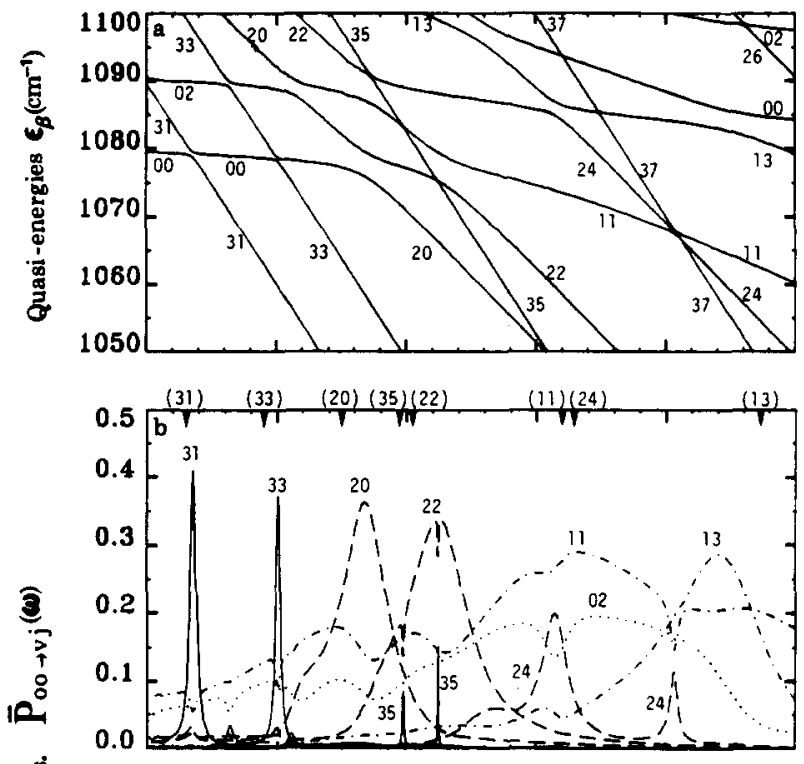



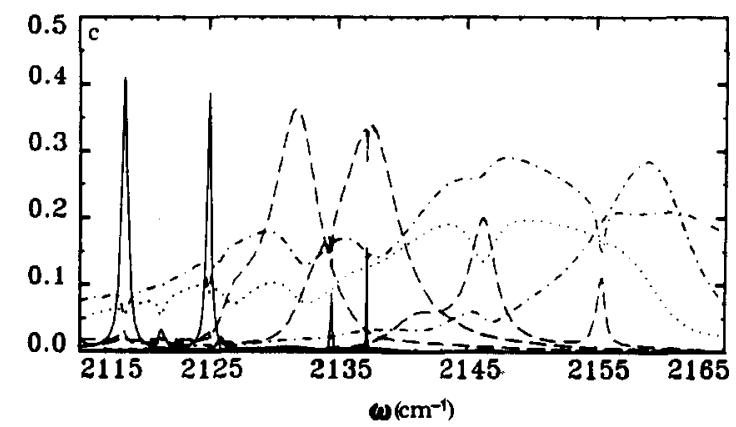

FIG. 7. (a) Frequency-dependent total quasienergies $\epsilon_{B}$ (denoted by $\beta=v j$ in the figure). (b) and (c) are time-averaged MPA transition probabilities $\bar{P}_{00 \rightarrow v j}$ for ${ }^{12} \mathrm{C}^{16} \mathrm{O}$ at $50 \mathrm{GW} / \mathrm{cm}^{2}$. Figures $7(a)$ and $7(b)$ are the results obtained by the nonadiabatic approximation, whereas Fig. 7 (c) is that of exact Floquet calculation. The triangles on the top of Fig. $7(b)$ indicate the zero-field transition energies. 

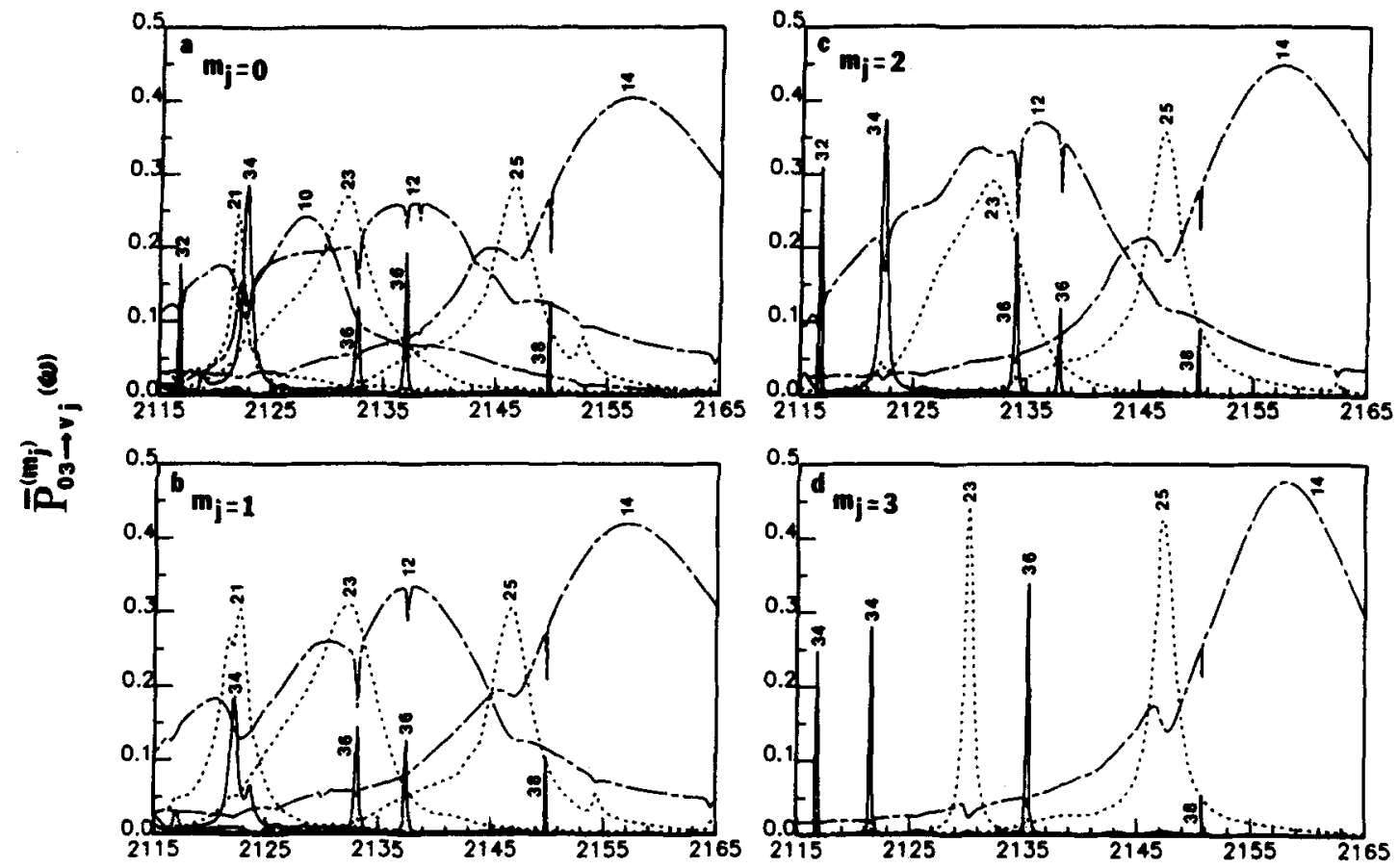

$\omega\left(\mathbf{c} \mathbf{m}^{-1}\right)$

FIG. 8. Time-averaged MPE transition probabilities for the diatomic molecule ${ }^{12} \mathrm{C}{ }^{16} \mathrm{O}$ initially prepared at states $\left|v=0, j=3, m_{j}\right\rangle$, with (a) $m_{j}=0$, (b) $m_{j}=1$, (c) $m_{j}=2$, and (d) $m_{j}=3$, respectively, at $50 \mathrm{GW} / \mathrm{cm}^{2}$. - - - , one-photon spectra; $\cdots$, two-photon spectra; and - three-photon spectra.

The general features of state-to-state MPA spectra are illustrated in Figs. 8(a) $-8(d)$ for molecules initially prepared in the states $\left|v=0, j=3, m_{j}\right\rangle$ with $m_{j}=0,1,2$ and 3 , respectively. Included within the frequency range of our interest are optically allowed direct absorption dominant channels

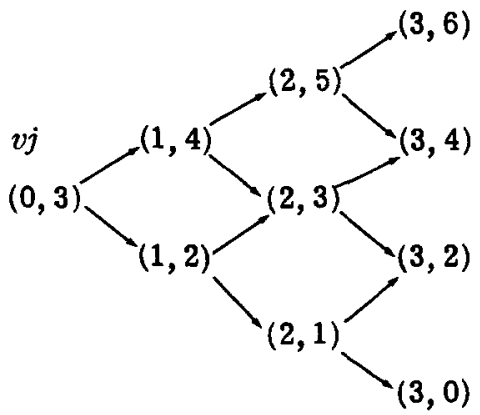

and two other higher order (stimulated emission and absorption occur simultaneously) dominant channels

$$
\begin{aligned}
& (0,3) \longrightarrow(1,2) \longrightarrow(2,1) \\
& (0,1) \longrightarrow(1,0),
\end{aligned}
$$

and

$$
\begin{aligned}
& (0,3) \longrightarrow(1,4) \longrightarrow(2,5) \longrightarrow(3,6) \longrightarrow(4,7) \\
& (0,5) \stackrel{\longrightarrow}{\longrightarrow}(1,6) \stackrel{\longrightarrow}{\longrightarrow}(2,7) \stackrel{\longrightarrow}{\longrightarrow}(3,8)^{\prime} .
\end{aligned}
$$

Besides the well-known nonlinear features, ${ }^{5}$ we found, in Figs. $8(\mathrm{a})-8(\mathrm{~d})$, a strong $m_{1}$ dependence of the MPA line shapes. In general, the power broadening effect is less prominent and the spectrum becomes less sophisticated as the quantum number $m_{j}$ is increased from 0 toward $j$ (here $j=3$ ). This is most evident when one compares Fig. $8(a)\left(m_{j}=0\right)$ with Fig. $8(d)\left(m_{j}=3\right)$. One can readily show that fewer channels exist for larger $m_{j}$ since the conservation of $m_{j}$ implies that only states with $j \geq\left|m_{j}\right|$ can participate in MPA process. The physics behind all these observations can be made clear by studying the adiabatic vibrational potentials such as those shown in Fig. 5. For a given $j$, molecules with larger $m_{f}$ tend to have more localized rotational wave functions and stay away from the laser field direction. This results in a reduction in the effective interaction between the molecule and the field and therefore the power broadening effect, etc. One notices that, in the classical limit, all MPA processes in the case of $m_{j}=j$ [Fig. 8(d)] will be totally quenched. Since the molecule will be then strictly confined in the plane perpendicular to the field direction, yielding vanishing molecule-field interaction. The $m_{j}$-averaged transition probabilities $\bar{P}_{03-v^{\circ} j^{\circ}, \text { Eq. }}(46)$, is displayed in Fig. 9. The spectral difference in Fig. $7(\mathrm{~b})\left(\bar{P}_{00-v^{\prime} y^{\prime}}\right)$ and Fig. $9\left(\bar{P}_{03-v^{\circ} y^{\prime}}\right)$ reflects the change in the initial rotational state $j$ as well as the $(2 j+1)$-degeneracy effect.

Finally, we show in Figs. 10(a) and 10(b) the calculated full MPA spectra [Eq. (47)] of ensembles of CO molecules at temperatures $T=10$ and $50 \mathrm{~K}$, respectively. In each figure, three distinct spectral profiles represent one- , two-, and three-vibrational quantum excitation, respectively. As can be seen, the singlequantum spectra $\bar{P}(v=0 \rightarrow v=1)$ are strongly power 


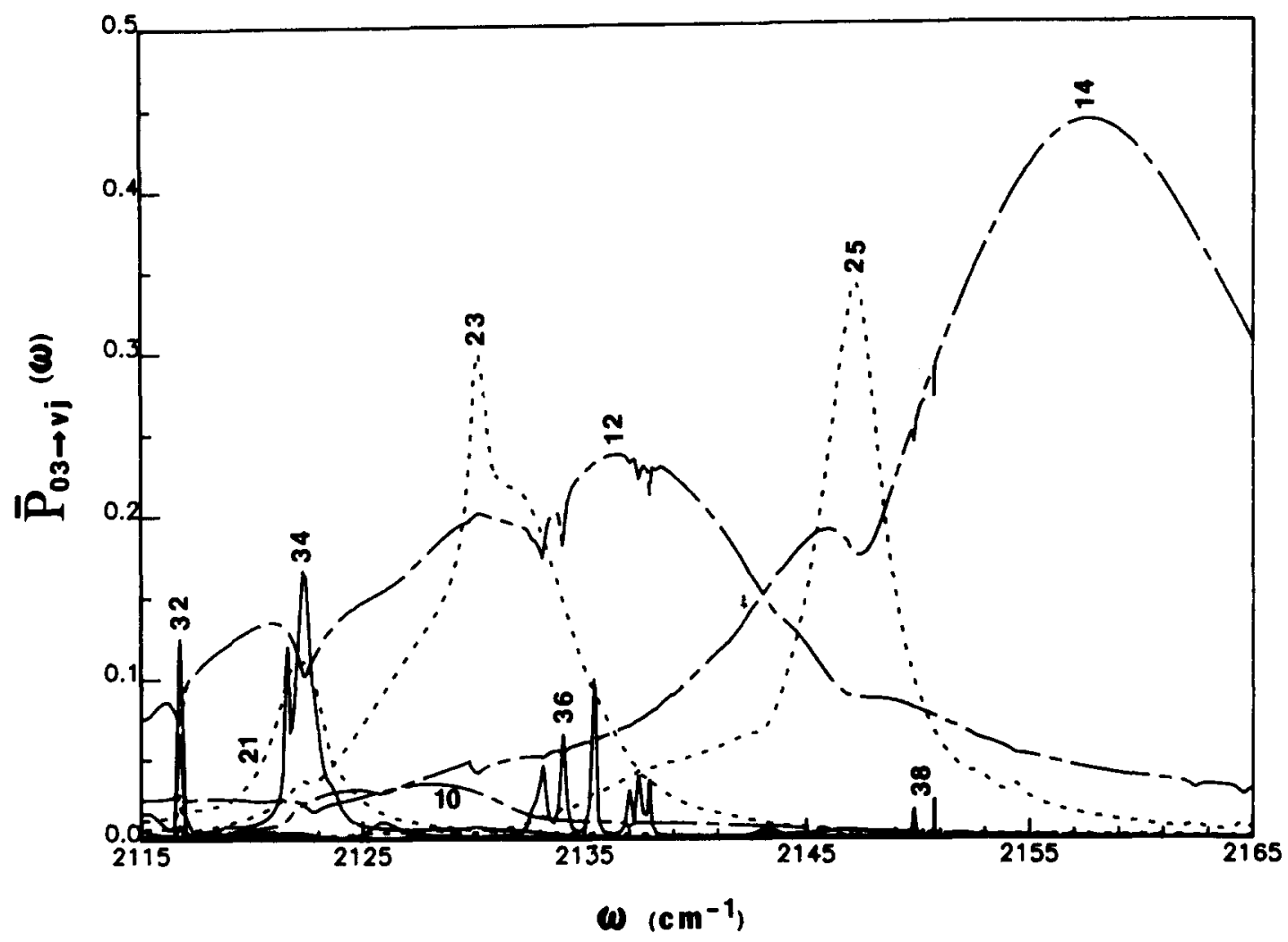

FIG. 9. Time and $m_{j}$-averaged MPE transition probabilities $\bar{P}_{03-v f}$ for the diatomic molecule ${ }^{12} \mathrm{C}^{16} \mathrm{O}$ at $50 \mathrm{GW} / \mathrm{cm}^{2}$. Curve notations same as Fig. 8 .

broadened to smear out the rotational structures. The double-quantum spectra $\bar{P}(v=0 \rightarrow v=2)$ are also substantially power broadened but still show some resolvable rotational structures. The triple-quantum spectra $\bar{P}(v=0-v=3)$ consist of mainly sharper and wellresolved spikes. As the temperature is increased, the initial rotational state distribution becomes more widely spread. This results in less defined and broader absorption profiles as can be seen in Fig. 10(b).

\section{CONCLUSIONS}

We have shown in this paper that the nonadiabatic approximation method provides a conceptually appealing and computationally powerful new approach for nonperturbative treatment of resonant MPA processes in intense fields. The utility of the method lies in its capability of generating economically accurate state-tostate MPA spectra which are essential for quantitative understanding of nonlinear MPE and MPD quantum dynamics. Further simplification of the nonadiabatic theory (and therefore more computational saving) are possible in several directions. For example, for heavy or large polyatomic molecules, the nonadiabatic coupling terms are expected to be much smaller than those of diatomic molecules and the adiabatic approximation may provide already an adequate description for MPA. Also in the case of weak to medium laser field strengths, the full vibrational-Floquet Hamiltonian analysis (Fig. 2) for QVE spectra may be unnecessary and can be re-
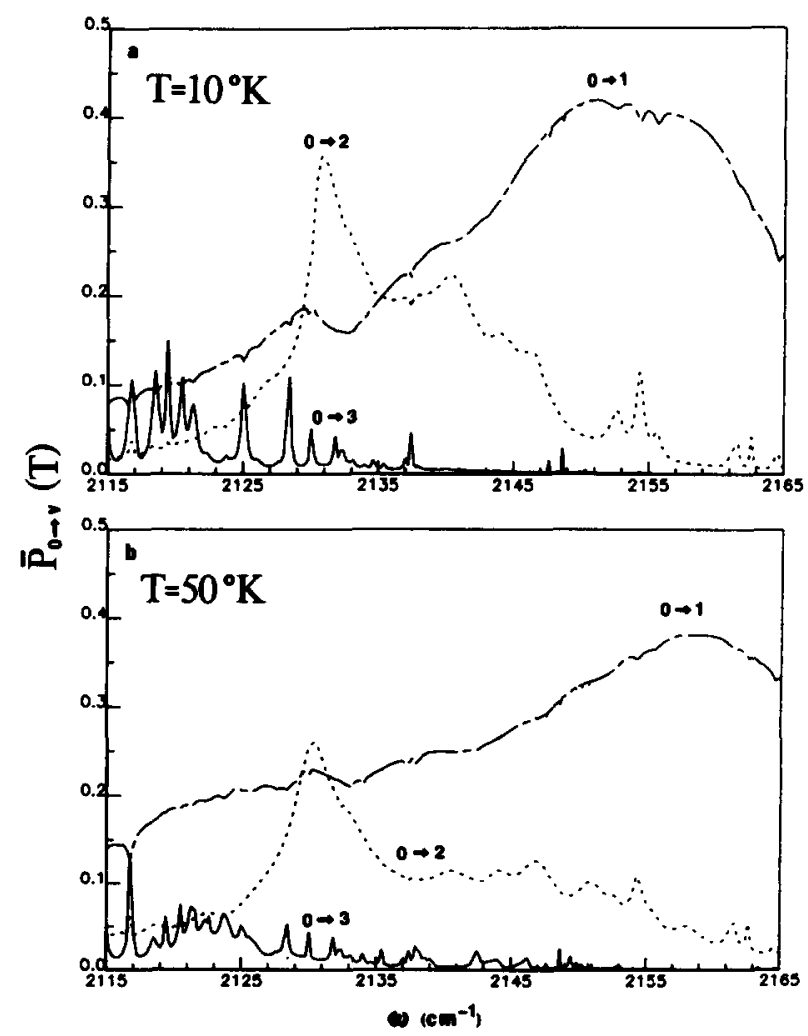

FIG. 10. Full MPA spectra of ensembles of ${ }^{12} \mathrm{C}^{16} \mathrm{O}$ molecules at temperatures (a) $T=10 \mathrm{~K}$ and (b) $T=50 \mathrm{~K}$, respectively. Curve notations same as Fig. 8. 
placed by simpler rotating wave approximation calculations. Extension of these ideas to the investigation of several polyatomic molecules $\left(\mathrm{SO}_{2}, \mathrm{O}_{3}, \mathrm{SF}_{6}\right)$ are underway. ${ }^{15}$

\section{ACKNOWLEDGMENTS}

This research was supported in part by the United States Department of Energy, Division of Chemical Sciences, under contract NO. DE-AC02-80ER10748 and by the Alfred P. Sloan Foundation. Acknowledgment is also made to the Donors of the Petroleum Research Fund, administered by the American Chemical Society, for partial support of this work. We are grateful to the United Telecom Computing Group (Kansas City) for generous support of the CRAY computer time.

${ }^{1}$ For recent reviews, see, for example, N. Bloembergen and E. Yablonovitch, Phys. Today 31, 23 (1978); P. A. Schulz, Aa. S. Suadbo, D. J. Krajnovich, H. S. Kwok, Y. R. Shen, and Y. T. Lee, Annu. Rev. Phys. Chem. 30, 379 (1979); Coherent Nonlinear Optics, edited by M. S. Feld and V. S. Letokhov (Springer, New York, 1980); V. S. Letokhov and A. A. Makarov, Sov. Phys. Usp. 241, 366 (1981).

${ }^{2} J$. H. Shirley, Phys. Rev. B 138, 979 (1965).

${ }^{3}$ D. R. Dion and J. O. Hirschfelder, Adv. Chem. Phys. 35, 265 (1976), and references therein.

${ }^{4}$ See, for example, J. V. Moloney and F. H. M. Faisal, J. Phys. B 12, 2829 (1979); S. I. Chu, Chem. Phys. Lett. 70,
205 (1980); S. C. Leasure, K. F. Milfeld, and R. E. Wyatt, J. Chem. Phys. 74, 6197 (1981).

${ }^{5}$ S. I. Chu, J. V. Tietz, and K. K. Datta, J. Chem. Phys. 77, 2968 (1982).

${ }^{6}$ See, for example, S. I. Chu, J. Chem. Phys. 75, 2215 (1981); S. I. Chu, C. Laughlin, and K. K. Datta, Chem. Phys. Lett. 98, 476 (1983).

${ }^{7}$ For recent reviews, see Atom-Molecule Collision Theory, edited by R. B. Bernstein (Plenum, New York, 1979).

${ }^{8}$ P. A. Braun and A. N. Petelin, Sov. Phys. JETP 39, 775 (1974); V. P. Makarov and M. V. Fedorov, ibid. 43, 615 (1976).

${ }^{9}$ S. I. Chu, T. S. Ho, and J. V. Tietz, Chem. Phys. Lett. (in press).

19Y. B. Zel'dovich, Sov. Phys. JETP 24, 1006 (1967); Ya. B. Zel'dovich, Sov. Phys. Usp. 16, 427 (1973); J. M. Okuniewicz, J. Math. Phys. 15, 1587 (1973); A. G. Fainshtein, N. L. Manakov, and L. P. Rapoport, J. Phys. B 11, 2561 (1978).

${ }^{11}$ By taking advantage of the fact that $\hat{H}_{R R}(\theta, \phi) P_{j}^{m f}(\cos \theta)$ $=B_{\theta} j(j+1) \hbar^{2} P_{j}^{m_{j}}(\operatorname{ocs} \theta)$ and expanding $\lambda_{\alpha}(\theta)$ and $F_{m, \alpha}(\theta)$ in terms of Legendre polynomials $P_{f}(\cos \theta)$, the $G$-matrix elements $\left\{g_{\alpha \alpha^{\circ}}^{f^{\prime}}\right\}$ can be evaluated efficiently and completely analytically in terms of $3 j$ symbols.

${ }^{12}$ The integral in Eq. (36) can be evaluated analytically if $F_{i m, \alpha}(\theta)$ are expanded in terms of $P_{f}(\cos \theta)$ as described in Ref. 11.

${ }^{13}$ A. W. Mantz, J. P. Maillard, W. B. Roh, and K. N. Rao, J. Mol. Spectrosc. 57, 155 (1975); W. Huo, J. Chem. Phys. 43, 624 (1965).

${ }^{14} \mathrm{~B}$. N. Parlett, The Symmetric Eigenvalue Problem (Prentice-Hall, Englewood Cliffs, New Jersey, 1980), Chap. 7.

${ }^{15} \mathrm{~J}$. V. Tietz and S. I. Chu (in preparation). 